\title{
Effect of double frequency heating on the lead afterglow beam currents of an electron cyclotron resonance ion source
}

\author{
V. Toivanen \\ Grand Accélérateur National d'Ions Lourds (GANIL), 14076 Caen Cedex 5, France \\ G. Bellodi, D. Küchler, and F. Wenander \\ European Organization for Nuclear Research (CERN), 1211 Geneva 23, Switzerland \\ O. Tarvainen \\ University of Jyväskylä, Department of Physics (JYFL), 40500 Jyväskylä, Finland
}

(Received 20 July 2017; published 4 October 2017)

\begin{abstract}
The effect of double frequency heating on the performance of the CERN GTS-LHC 14.5 GHz Electron Cyclotron Resonance (ECR) ion source in afterglow mode is reported. The source of the secondary microwave frequency was operated both in pulsed and continuous wave $(\mathrm{CW})$ modes within the range of 12-18 GHz. The results demonstrate that the addition of the secondary frequency can significantly impact the extracted beam currents and the temporal stability of the beam during the afterglow discharge. For example, up to a factor of 2.6 increase was achieved for ${ }^{208} \mathrm{~Pb}^{35+}$ and a factor of 3.1 for ${ }^{208} \mathrm{~Pb}^{37+}$ compared to single frequency afterglow currents. It is shown that these effects are dependent on the choice of the secondary frequency with respect to the primary one and on the temporal synchronization between the two microwave sources. Overall, the results provide new insight into the afterglow discharge supporting the prevailing understanding of the physical processes behind the phenomenon.
\end{abstract}

DOI: 10.1103/PhysRevAccelBeams.20.103402

\section{INTRODUCTION}

Thanks to their reliability in long term operation and capability to produce intense beams of multiply charged heavy ions from a wide selection of gaseous and solid elements, Electron Cyclotron Resonance (ECR) ion sources [1] are employed in a broad range of research and industrial applications. At the European Organization for Nuclear Research (CERN) the GTS-LHC $14.5 \mathrm{GHz}$ ECR ion source is used to produce pulsed heavy ion beams for the CERN experimental program, including collider experiments with the Large Hadron Collider (LHC) and fixed target physics studies with the Super Proton Synchrotron (SPS) [2,3]. Following the advancement of the experiments, the requirements for the primary ion beams are constantly increasing. In order to meet the conditions for future operation, e.g., at CERN, continued ion source development remains essential.

Over the last decades, a selection of methods have been developed to enhance the performance of ECR ion sources, especially in terms of the beam currents of highly charged ions [4]. These include, for example, the simultaneous

\footnotetext{
*ville.toivanen@ganil.fr
}

Published by the American Physical Society under the terms of the Creative Commons Attribution 4.0 International license. Further distribution of this work must maintain attribution to the author(s) and the published article's title, journal citation, and DOI. injection of light and heavy gas species into the ECR plasma to boost the extracted currents of the heavier component (also known as gas mixing), the installation of a biased electrode at the injection end of the plasma chamber to mitigate electron losses and consequently increase the plasma electron density, and the choice of plasma chamber materials to increase secondary electron production. These methods are currently utilized in most ECR ion sources operated around the world. Another well-established technique is to deliver the microwave power-which sustains the plasma and the ionization processes-with two (or more) discrete frequencies yielding an improvement in the beam currents of highly charged ions [5]. This effect is usually attributed to the creation of a second electron cyclotron resonance surface inside the plasma volume, which presumably affects the energy transfer from the microwaves to the plasma electron population and thereby modifies the plasma properties favorably for the high charge state ion production. Moreover, it has been shown that adjusting the secondary frequency as well as the power balance between the two frequencies serves as a means to optimize the ion source performance [6].

The techniques discussed above have been discovered and are mostly utilized in ECR ion sources operated in $\mathrm{CW}$ (continuous wave) mode, in which the microwave power delivery to the source is continuous, resulting in the extraction of a continuous ion beam. Pulsed beam operation is most commonly achieved by pulsing the microwave 
power. In this operation mode the extracted beam currents commonly exhibit an afterglow transient [7], which is characterized by an intense burst of highly charged ions following the switch-off of the microwave power. During the afterglow, usually lasting for a few milliseconds, the extracted beam currents can be several times higher than during the actual microwave injection. This property makes the afterglow operation a tempting option for applications requiring intense but short pulses of highly charged ions, as it offers access to higher beam currents than is otherwise available from the ion source. The afterglow process is discussed in more detail in the next chapter.

The afterglow operation can be combined with the other methods described above to further improve the beam performance. However, the combination of double frequency heating and afterglow has not been widely studied. Furthermore, it was shown in a recent study, performed with single frequency heating, that the choice of the microwave frequency does impact the afterglow performance [8]. These results suggest that applying double frequency heating could yield additional performance improvements, providing a clear motivation for further studies.

In this paper the combination of the afterglow operation and the double frequency heating is studied in detail with the CERN GTS-LHC ECR ion source. It provides an excellent platform for the experiments, as it is one of the few ECR ion sources in the world routinely operated in afterglow mode. In addition, it employs most of the standard methods to improve beam performance, such as gas mixing and the biased disc. The emphasis of the presented experiments is to study the influence of the secondary frequency on the extracted afterglow currents and charge state distributions, in a wide $12-18 \mathrm{GHz}$ range around the primary frequency of $14.5 \mathrm{GHz}$. The experiments include both $\mathrm{CW}$ and pulsed operation of the secondary frequency and the effects of varying the pulse timing between the two microwave sources are presented.

\section{EXPERIMENTAL SETUP AND PROCEDURE}

The experimental work described in this paper was performed with the GTS-LHC $14.5 \mathrm{GHz}$ ECR ion source [9]. GTS-LHC is a second generation room-temperature ECR ion source based on the design of the Grenoble Test Source (GTS) by CEA Grenoble (Commissariat à l'Énergie Atomique) [10]. It features three solenoids and a 36-piece Halbach-style hexapole for magnetic confinement of the plasma, axial microwave injection into the plasma chamber with rectangular wave guides, and a three electrode beam extraction system. The ion source is used mainly for lead beam production relying on two resistively heated miniature ovens evaporating lead into the plasma chamber. The predominant ion species produced for the CERN experimental heavy ion program is ${ }^{208} \mathrm{~Pb}^{29+}$. Further information about the features and operation of GTS-LHC is presented, e.g., in Refs. $[9,11,12]$.
GTS-LHC is operated almost exclusively in afterglow mode by pulsing the injected microwaves at $10 \mathrm{~Hz}$ with $50 \%$ duty factor, resulting in a delivered microwave pulse length of $50 \mathrm{~ms}$. The afterglow discharge is characterized by an abrupt loss of the cold electron population due to the lack of ECR heating and resulting confinement. The surge of cold electrons escaping the magnetic trap is accompanied by increased loss rate of the positive ions expelled from the plasma by the ambipolar potential. As a result, an intense burst of highly charged ions, lasting for a few milliseconds, is exhibited by the extracted ion beam current. The peak beam currents of the highly charged ions can be several times higher during the afterglow burst than the steady state phase of the operation. A comprehensive description of the afterglow discharge is presented e.g., in Ref. [13] and in the references therein.

In order to maximize the ion beam currents during the afterglow, a significantly different ion source tuning is usually required compared to the operation in $\mathrm{CW}$ mode. In the case of the GTS-LHC one of the main differences is the stronger magnetic field than what is conventionally suggested by the semiempirical scaling laws derived for efficient beam production with ECR ion sources. The local field maxima proposed by the scaling laws at the injection and extraction boundaries of the plasma chamber, as well as the minimum field near the center of the chamber, can be presented with the electron cyclotron resonance field $B_{\mathrm{ECR}}$ as [14]:

$$
\begin{aligned}
& B_{\mathrm{inj}}=4 B_{\mathrm{ECR}}, \\
& B_{\mathrm{ext}}=1.8 B_{\mathrm{ECR}} \quad \text { and } \quad B_{\text {min }}=0.8 B_{\mathrm{ECR}}
\end{aligned}
$$

For a $14.5 \mathrm{GHz}$ ECR ion source the resonance field is $B_{\mathrm{ECR}}=0.52 \mathrm{~T}$, which gives a magnetic field scaling of $B_{\text {inj }}=2.08 \mathrm{~T}, B_{\text {ext }}=0.94 \mathrm{~T}$, and $B_{\min }=0.42 \mathrm{~T}$. However, in order to achieve the optimum performance in afterglow, the GTS-LHC is normally operated with values around $B_{\text {inj }}=2.22 \mathrm{~T}, B_{\text {ext }}=1.38 \mathrm{~T}$, and $B_{\min }=0.48 \mathrm{~T}$, resulting in significantly higher mirror ratios than normally used in $\mathrm{CW}$ operation mode. The axial $B$ field profile is presented in Fig. 1. The fields have been calculated with a 3D magnetic model of GTS-LHC which has been validated with magnetic field measurements.

A possible explanation for the requirement of higher $B$ fields comes from the difference in the ion extraction process from the plasma between $\mathrm{CW}$ and afterglow operation. In $\mathrm{CW}$ mode the only process to extract ions from the plasma is the steady flux of ions that escape the confinement. As such the optimization of extracted beam current of multiply charged ions leads to a compromise between confinement and losses. Optimizing the extracted currents of high charge state ions which can be viewed as a favorable loss process sets an upper limit for the mirror ratios. In afterglow mode it is preferable to mitigate the ion losses during the rf pulse as much as possible to maximize 


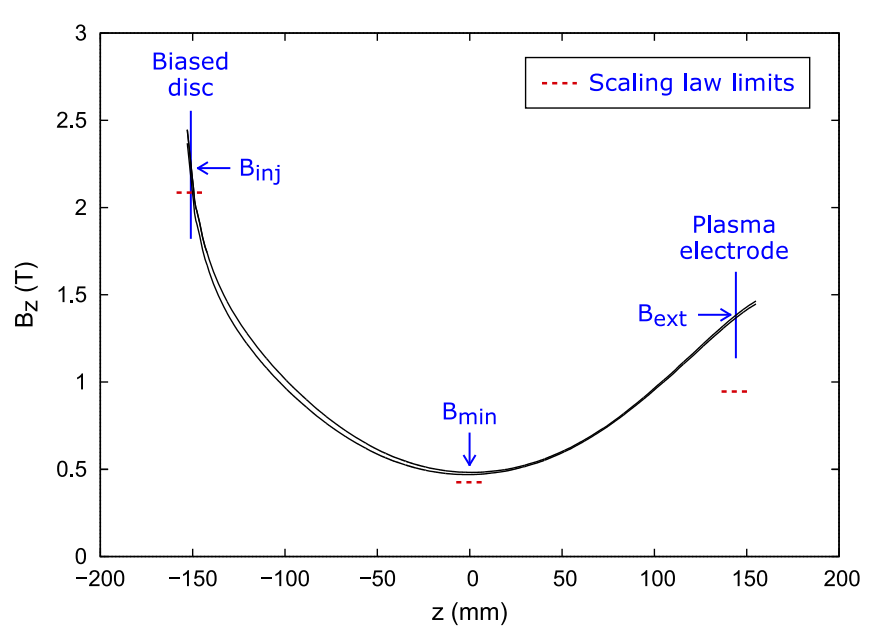

FIG. 1. The on-axis magnetic field of the GTS-LHC and the conventional scaling law limits for $14.5 \mathrm{GHz}$ ECRIS (dashed lines). The resonance field for $14.5 \mathrm{GHz}$ is $B_{\mathrm{ECR}}=0.52 \mathrm{~T}$. The axial field variation due to different solenoid settings used in this work falls within the presented range of field strength. The biased disc and the plasma electrode locations represent the axial boundaries of the plasma chamber.

the extracted current when the ions are released from the trap following the rf switch-off. This action is achieved by increasing the mirror ratios. Furthermore, recent ECR plasma studies have shown that increasing the field strength yields higher extracted beam currents of highly charged ions, up to the point where kinetic instabilities are triggered in the plasma, which leads to a drastic decrease in the performance [15]. The threshold magnetic field strength for the appearance of the instabilities can be characterized with the $B_{\min } / B_{\mathrm{ECR}}$ ratio. Depending on the ion source properties, the threshold has been shown to be in the range of 0.7-0.8. In the case of GTS-LHC, the optimum performance is reached with $B_{\min } / B_{\mathrm{ECR}}>0.9$, which is significantly higher than the aforementioned stability limit. In Ref. [16] it is shown that the time scales which are required for the onset of the kinetic instabilities following the plasma ignition are on the order of a few tens to a few hundreds of milliseconds, depending on the parameter tuning of the ion source. This suggests that with the nominal tuning of GTS-LHC for afterglow operation, the rf pulse length of $50 \mathrm{~ms}$ is shorter than the time required for the instabilities to be triggered, which allows the use of higher field values than is normally available in CW operation to optimize the extracted beam currents in afterglow.

The GTS-LHC features two WR62 type rectangular wave guides for microwave injection into the plasma chamber. Both wave guides are inserted axially through the ion source injection plug and enter the plasma chamber off-center and symmetrically from both sides of the biased disc. The arrangement of the wave guides and the biased disc at the plasma chamber injection end is presented in Fig. 2. The plasma chamber is $295 \mathrm{~mm}$ long (from the

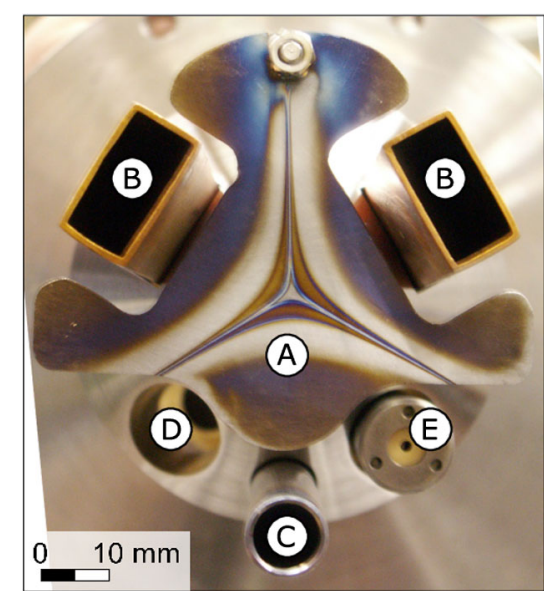

FIG. 2. The GTS-LHC ECR ion source injection plug, viewed from the plasma chamber. At the center is the biased disc (A), which wraps around the two WR62 type rectangular wave guides (B), seen in the figure on top left and right. The gas inlet is at the bottom (C), surrounded on both sides by the two circular oven ports, one shown empty (D) and the other with the oven in place (E).

biased disc to the $\phi=12 \mathrm{~mm}$ beam extraction aperture) and $78 \mathrm{~mm}$ in diameter. It is made of stainless steel for enhanced durability in long term pulsed operation. In normal operation the wave guides are connected to two klystrons, both providing microwaves at $14.5 \mathrm{GHz}$ frequency. One of the klystrons is used for operation and the second one serves as a spare unit to minimize operation down time in the case of klystron malfunction. Both wave guides are equipped with a high voltage break and a vacuum window.

In the double frequency heating experiments the wave guide of the spare klystron was connected to an Amplifier Research model 300T7z5G18 traveling wave tube amplifier (TWTA). The unit is capable of amplifying microwave radiation in the frequency range of $7.5-18 \mathrm{GHz}$ to at least $300 \mathrm{~W}$ of power (the actual maximum varies between 300 and $400 \mathrm{~W}$ depending on the frequency). The transmission and reflection properties of the high voltage break and the vacuum window were measured as a function of the microwave frequency with a network analyser to determine the frequency range that can be used with the TWTA without significant power losses. The cutoff frequency of WR62 wave guide itself is $9.49 \mathrm{GHz}$ and the attenuation increases significantly before this limit is reached. As a result, the secondary frequency range used in the experiments was limited between 12 and $18 \mathrm{GHz}$, the lower limit being set by the properties of the high voltage break. An isolator element, i.e. a circulator with a load, which allows microwaves to pass through only in one direction was placed at the output of the TWTA to protect it from the primary $14.5 \mathrm{GHz}$ microwave radiation. The input signal for the TWTA was supplied from an HP model 8341B 


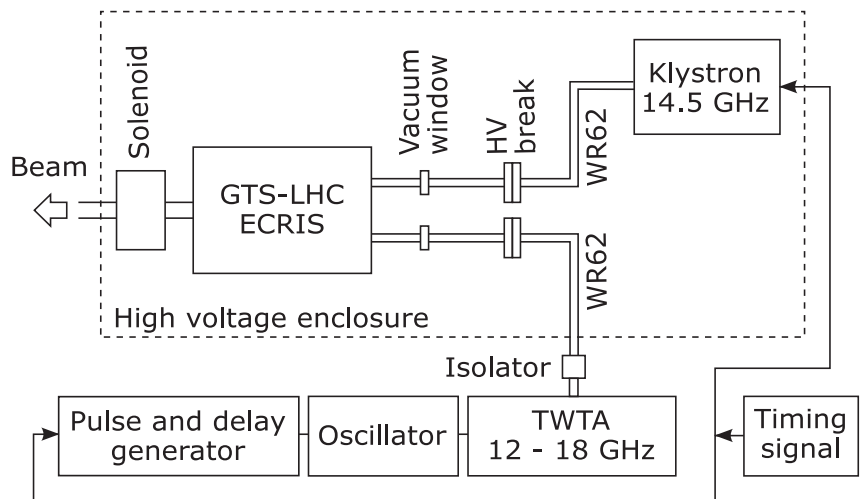

FIG. 3. A schematic presentation of the measurement setup used in the double frequency heating experiments.

variable frequency oscillator. A digital delay and pulse generator, SRS model DG535, was used to modulate the oscillator signal to allow pulsed TWTA operation. The timing signal driving the klystron pulsed operation was provided as a triggering signal to the pulse generator to allow the TWTA pulse pattern to be adjusted with respect to the klystron rf pulse. A schematic of the experimental setup is presented in Fig. 3 .

The experiments presented in this paper were performed using lead ion beams produced from metallic isotope purified ${ }^{208} \mathrm{~Pb}$ samples. The lead was evaporated into the ion source plasma chamber with two resistively heated miniature ovens and ionized in a plasma sustained by oxygen gas injection. The ion beam was extracted with $18.8 \mathrm{kV}$. In the experiments the ion source tuning was initially optimized for the production of ${ }^{208} \mathrm{~Pb}^{29+}$ ion beam using $\sim 1.9 \mathrm{~kW}$ of pulsed $14.5 \mathrm{GHz}$ microwaves from the klystron at $10 \mathrm{~Hz}$ and $50 \%$ duty cycle. The effects of the double frequency plasma heating on the afterglow beam performance were studied by operating the TWTA in both $\mathrm{CW}$ and pulsed modes with varied frequency. In the pulsed operation the TWTA and klystron pulses were first synchronized, and the TWTA pulse length and the delay between the trailing edges of the klystron and the TWTA rf pulses were varied. In addition, the effects of varying the power balance between the two microwave sources were studied.

The influence of the double frequency heating to the ion source performance during the afterglow burst was quantified with time-resolved beam current measurements using a Faraday cup in the low energy beam transport (LEBT) section of Linac3. Figure 4 presents the LEBT layout and the locations of the main ion optical elements and the Faraday cup.

In normal operation a $200 \mu$ s section of the afterglow burst is finally accelerated through Linac3, and consequently the majority of the beam pulse extracted from the ion source is lost inside the machine. In order to prevent the beam losses from causing interference with the linac rf

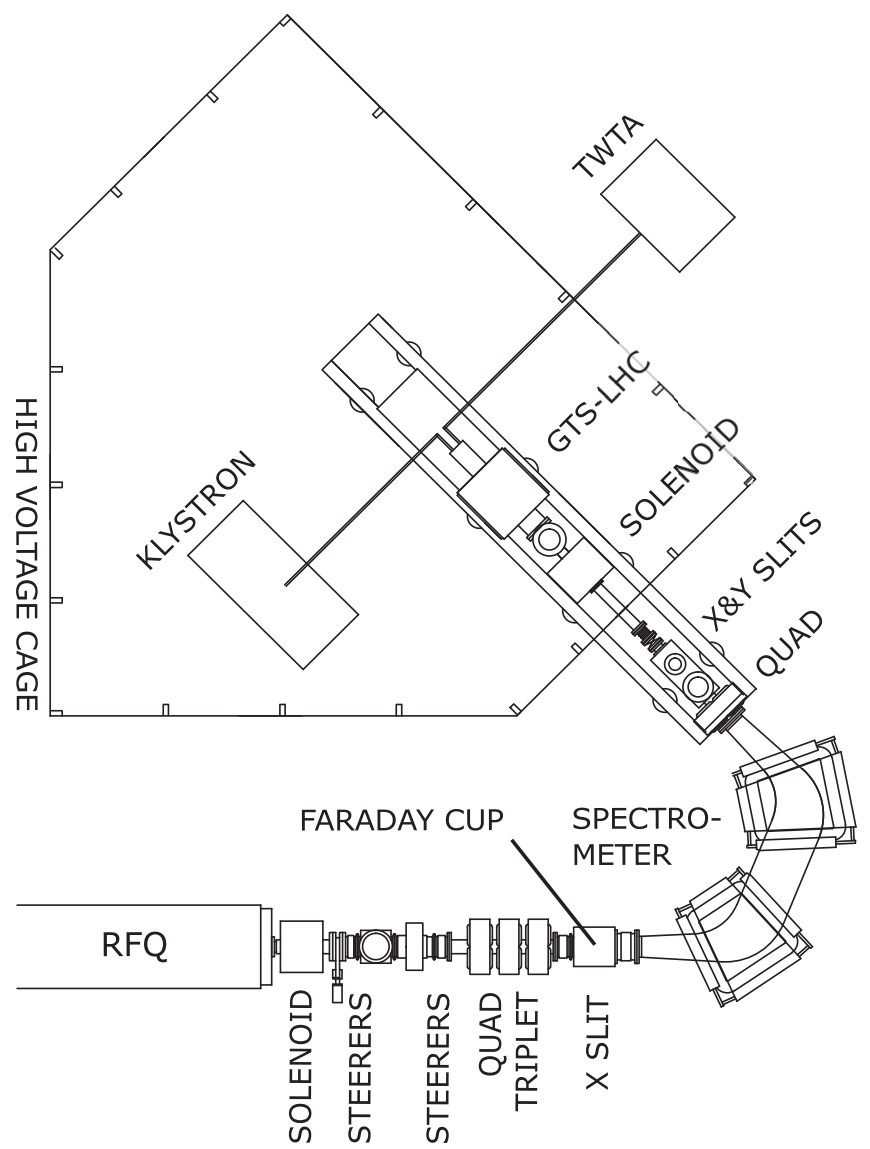

FIG. 4. Layout of Linac3 low energy beam transport section, from the ion source to the entrance of the RFQ. The main ion optical elements and beam diagnostics are also indicated.

systems, it is desirable to dump the unwanted parts of the beam pulse in the low energy beam transport section. In order to do this, a $1.3 \mathrm{~ms}$ long step-like modulation of $250 \mathrm{~V}$ is applied on top of the ion source extraction voltage during the beginning of the afterglow burst. As a result, the steady state section of the beam and the tail of the afterglow are shifted in energy compared to the peak of the afterglow, and are consequently lost in the spectrometer magnet downstream from the ion source. The effect of the modulation on the measured beam current after the spectrometer is presented in Fig. 5.

At the beginning of the afterglow burst the extracted beam current typically exhibits a period of instability that can last for several hundreds of microseconds (see Fig. 5). This behavior is likely caused by kinetic plasma instabilities which are triggered by the uneven collapse of the electron energy distribution function following the $\mathrm{rf}$ switch-off resulting in an excess of hot electrons, as discussed in Refs. [17-19]. The beam current behavior during the instability period typically varies from one beam pulse to the next. However, after the instabilities the current behavior exhibits a good pulse-to-pulse repeatability. 


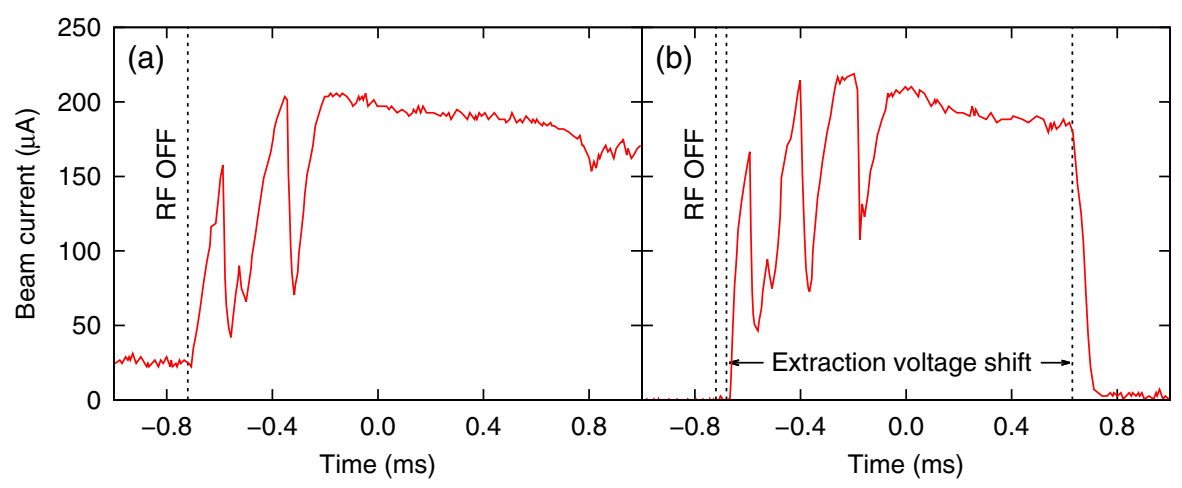

FIG. 5. The effect of the GTS-LHC extraction voltage modulation on the measured ${ }^{208} \mathrm{~Pb}^{29+}$ beam current during the afterglow. In case (a) the modulation is not applied and consequently the steady state beam current at the end of the klystron rf pulse is observed $(t<-0.72 \mathrm{~ms})$, and the afterglow current continues until the end of the measured time window. In case (b) the modulation is used and these parts are discarded before the beam reaches the Faraday cup.

\section{EXPERIMENTAL RESULTS}

Two cases were studied with varied pulse pattern and timing of the secondary microwaves with respect to the primary microwave pulse. First, the secondary microwave source was set to $\mathrm{CW}$ mode, providing continuous microwave injection into the plasma chamber. Second, the secondary microwaves were applied with pulse lengths comparable to the primary microwaves. The two microwave pulses were first synchronized by overlapping them temporally, after which the delay between the primary and the secondary microwave pulse trailing edges was varied. In both cases the primary $14.50 \mathrm{GHz}$ microwave pulse pattern was kept constant at $10 \mathrm{~Hz}, 50 \%$ duty cycle.

\section{A. CW operation of the secondary frequency}

Operating the TWTA in CW mode while pulsing the main frequency leads to a clear decrease in the afterglow beam current compared to the nominal single frequency operation. This is demonstrated in Fig. 6, which presents the afterglow beam currents of $\mathrm{Pb}^{29+}$ and $\mathrm{Pb}^{35+}$ as a function of the secondary frequency. It was observed that the low and medium charge states (represented by the
$\mathrm{Pb}^{29+}$ i.e. the peak of the charge state distribution) always decrease when the secondary frequency is applied. In general the same is also observed with the high charge states, as is demonstrated by $\mathrm{Pb}^{35+}$ in Fig. 6. The beam currents of the high charge states exhibit an increasing trend with decreasing frequency of the secondary microwave source. In the case of $\mathrm{Pb}^{35+}$ this actually leads to modest improvement in the measured beam current at the lowest secondary frequencies compared to the nominal operation [see Fig. 6(b)].

Figure 7 presents the beam current evolution during the afterglow discharge with selected secondary frequencies compared to the nominal single frequency operation. Apart from the variation of the current level during the afterglow, as discussed above, it was also observed that the beam stability during the afterglow is influenced by the frequency. If the secondary frequency was chosen to be higher than the primary one, the beam current instabilities that are normally seen at the beginning of the afterglow burst disappeared. With frequencies below the primary one this effect was not observed. Instead, with some of the frequencies the instabilities were even further amplified. This phenomenon is shown in Fig. 7(b).

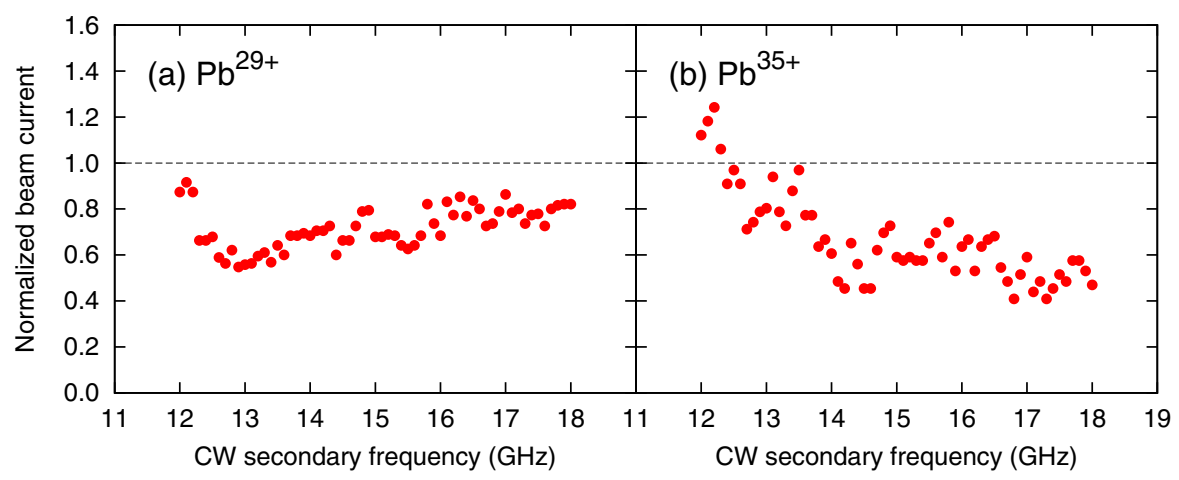

FIG. 6. $\mathrm{Pb}^{29+}$ and $\mathrm{Pb}^{35+}$ afterglow beam currents as a function of the secondary frequency operated in CW mode normalized to the single frequency beam currents $\left(190 \mu \mathrm{A}\right.$ for $\mathrm{Pb}^{29+}$ and $33 \mu \mathrm{A}$ for $\mathrm{Pb}^{35+}$ ). The main frequency of $14.5 \mathrm{GHz}$ is pulsed with $1920 \mathrm{~W}$ and the secondary frequency is operated at a constant $200 \mathrm{~W}$ power. 


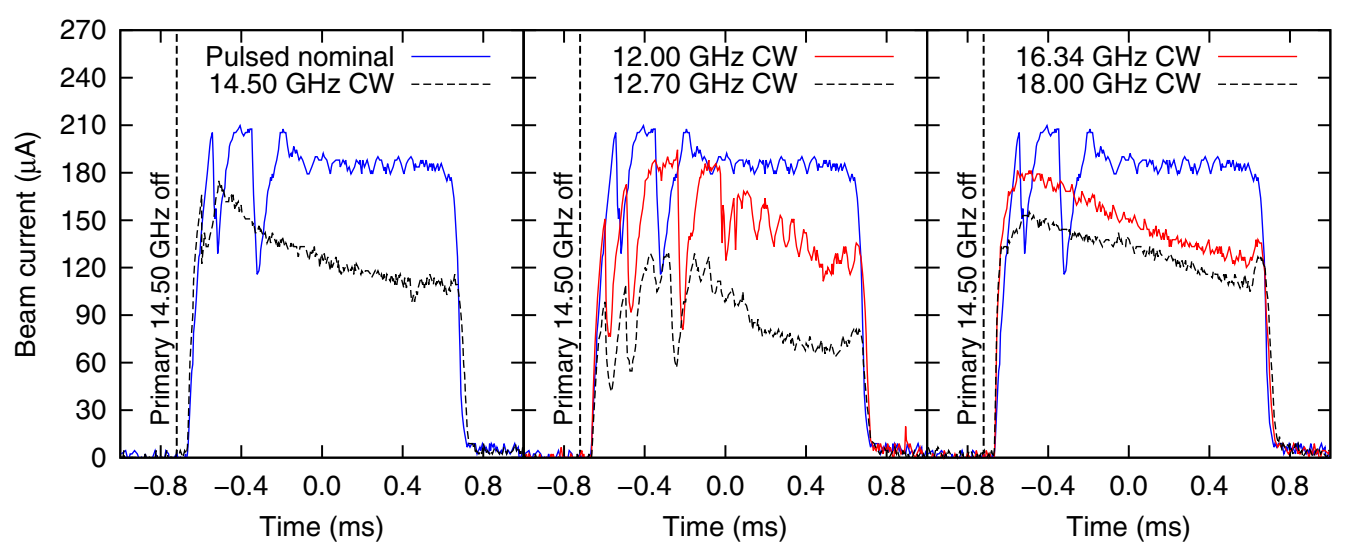

FIG. 7. ${ }^{208} \mathrm{~Pb}^{29+}$ beam current during the afterglow with pulsed primary and CW secondary frequency. The nominal single frequency case ("Pulsed nominal") is presented for comparison. The primary $14.50 \mathrm{GHz}$ frequency is supplied at $1930 \mathrm{~W}$ in all cases. The secondary frequency powers for $12.00,12.70,14.50,16.34$, and $18.00 \mathrm{GHz}$ are 161, 204, 200, 175, and $200 \mathrm{~W}$, respectively.

Figure 8 presents lead charge state distributions (CSDs) measured with two different secondary frequencies in $\mathrm{CW}$ and compared to a distribution measured with the nominal

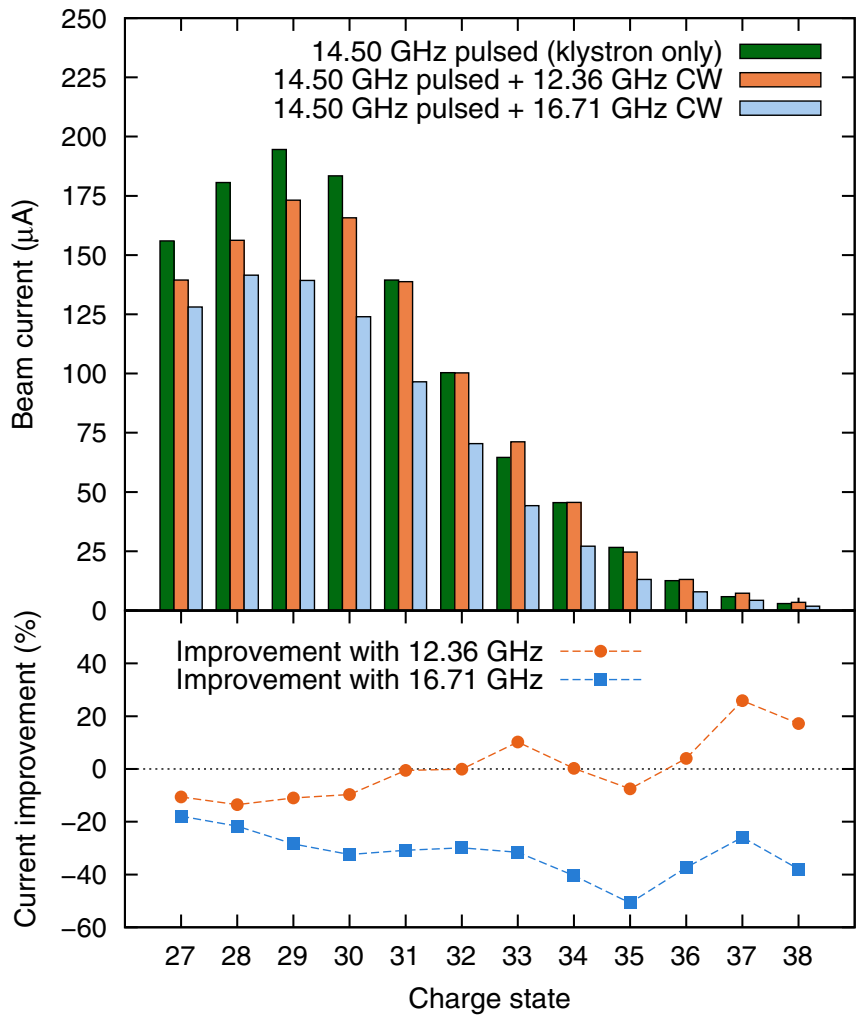

FIG. 8. Lead charge state distributions measured during afterglow with pulsed main frequency mixed with a secondary frequency operated in $\mathrm{CW}$ mode. Primary frequency operated at $1920 \mathrm{~W}$ and the secondary at $200 \mathrm{~W}$. Three cases are presented, (1) pulsed primary frequency only at $14.50 \mathrm{GHz}$, (2) pulsed primary at $14.50 \mathrm{GHz}$ with $12.36 \mathrm{GHz}$ secondary in $\mathrm{CW}$, and (3) pulsed primary at $14.50 \mathrm{GHz}$ with $16.71 \mathrm{GHz}$ secondary in $\mathrm{CW}$. In addition the change in the beam currents of different charge states with added secondary frequency is presented (points connected with dashed lines). (pulsed) single frequency operation conditions. The secondary frequencies were chosen so that one is significantly lower than the primary frequency and the other significantly higher. It is seen that with the higher secondary frequency of $16.71 \mathrm{GHz}$ the beam currents of all measure charge states are reduced compared to single frequency operation. The same is observed with the lower frequency of $12.36 \mathrm{GHz}$, apart from a few of the highest charge states which exhibit increased beam currents.

\section{B. Pulsed operation of the secondary frequency}

For the pulsed operation both the primary and the secondary microwave pulses were first synchronized and applied at identical pulse lengths and repetition rates $(50 \mathrm{~ms}$ and $10 \mathrm{~Hz}$ in this case). In this mode the secondary microwave radiation has a clear frequency and charge state dependent effect on the extracted beam currents. Figure 9 presents the measured beam currents of $\mathrm{Pb}^{29+}$ and $\mathrm{Pb}^{35+}$ with varied secondary frequency and constant TWTA power of $200 \mathrm{~W}$. It is observed that the varied frequency has very weak effect on the $\mathrm{Pb}^{29+}$, whereas the $\mathrm{Pb}^{35+}$ current exhibits significant increases of up to a factor of 2.6 compared to the nominal single frequency operation. It is seen that the greatest improvements are achieved with frequencies below the primary microwave frequency of $14.5 \mathrm{GHz}$. With higher frequencies the improvements decrease with increasing frequency and ultimately disappear.

The frequency dependent effect is further demonstrated in Fig. 10 which presents the lead charge state distributions measured with two secondary frequencies while both microwave sources are operated in pulsed mode. With a secondary frequency of $12.36 \mathrm{GHz}$ (below the primary frequency) the ion currents of the low lead charge states (below the distribution peak) decrease, while the currents of the highest charge states increase. As a result, the peak of the distribution shifts one charge state higher compared to the single frequency operation. When the secondary 


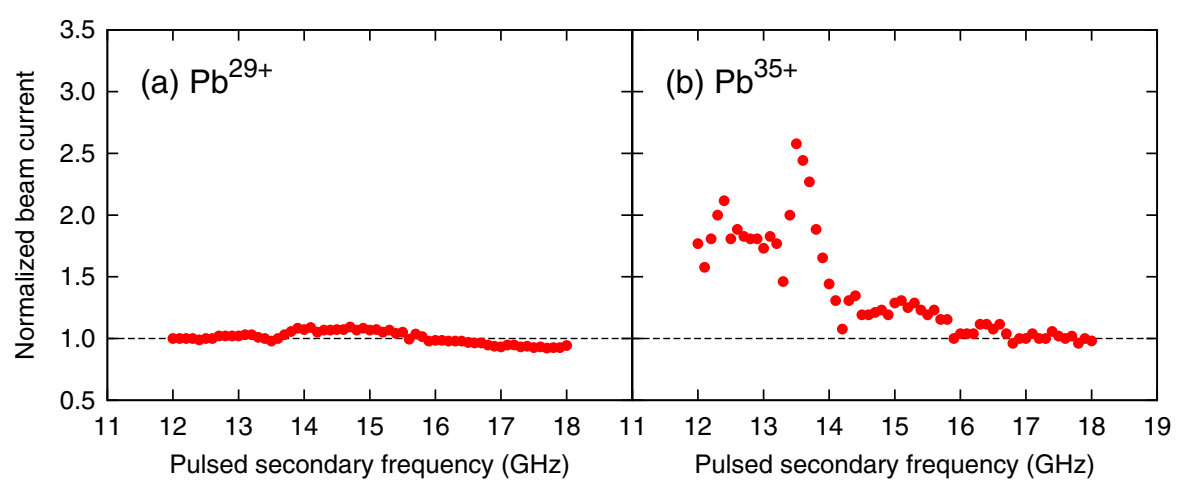

FIG. 9. $\mathrm{Pb}^{29+}$ and $\mathrm{Pb}^{35+}$ afterglow beam currents as a function of the secondary frequency operated in pulsed mode normalized to the single frequency beam currents (192 $\mu \mathrm{A}$ for $\mathrm{Pb}^{29+}$ and $26 \mu \mathrm{A}$ for $\mathrm{Pb}^{35+}$ ). The main frequency of $14.5 \mathrm{GHz}$ is pulsed with $1920 \mathrm{~W}$ and the secondary frequency is operated at a constant $200 \mathrm{~W}$ power.

frequency is higher than the primary one, a significantly weaker influence on the beam currents and CSD is seen.

In order to verify that the effects seen in the CSD are not caused by increased total microwave power, the lead charge

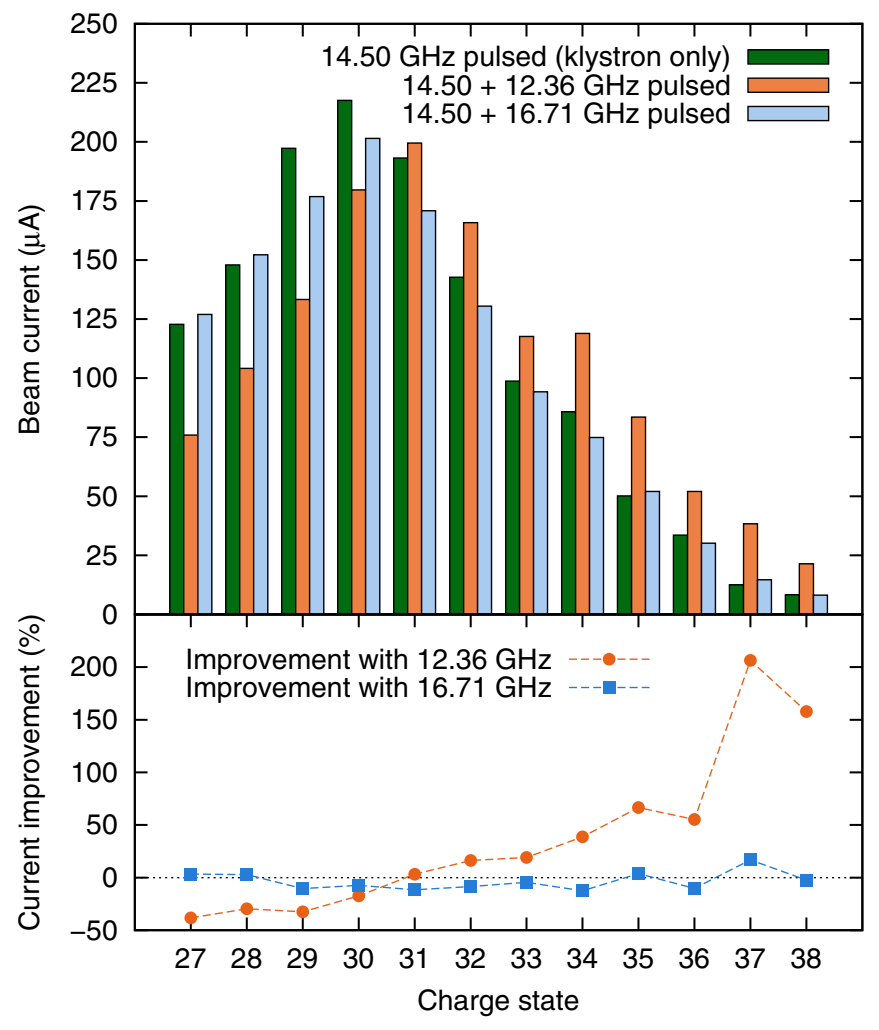

FIG. 10. Lead charge state distribution during afterglow with two different secondary microwave frequencies. Klystron operated at $1920 \mathrm{~W}$, TWTA at 180 and $185 \mathrm{~W}$ for 12.36 and 16.71 GHz, respectively. Both microwave sources are pulsed at $10 \mathrm{~Hz}$. Three cases are presented, (1) klystron only at $14.50 \mathrm{GHz}$, (2) klystron at $14.50 \mathrm{GHz}$ with $12.36 \mathrm{GHz}$ secondary frequency from TWTA, and (3) klystron at $14.50 \mathrm{GHz}$ with $16.71 \mathrm{GHz}$ secondary frequency. In addition the change in the beam current of different charge states with secondary frequency is presented (points connected with dashed lines). state distributions were measured under three conditions: (1) single frequency operation with nominal power of $1920 \mathrm{~W}$, (2) primary frequency at $1730 \mathrm{~W}$ and secondary frequency of $12.36 \mathrm{GHz}$ at $190 \mathrm{~W}$, summing up to the same

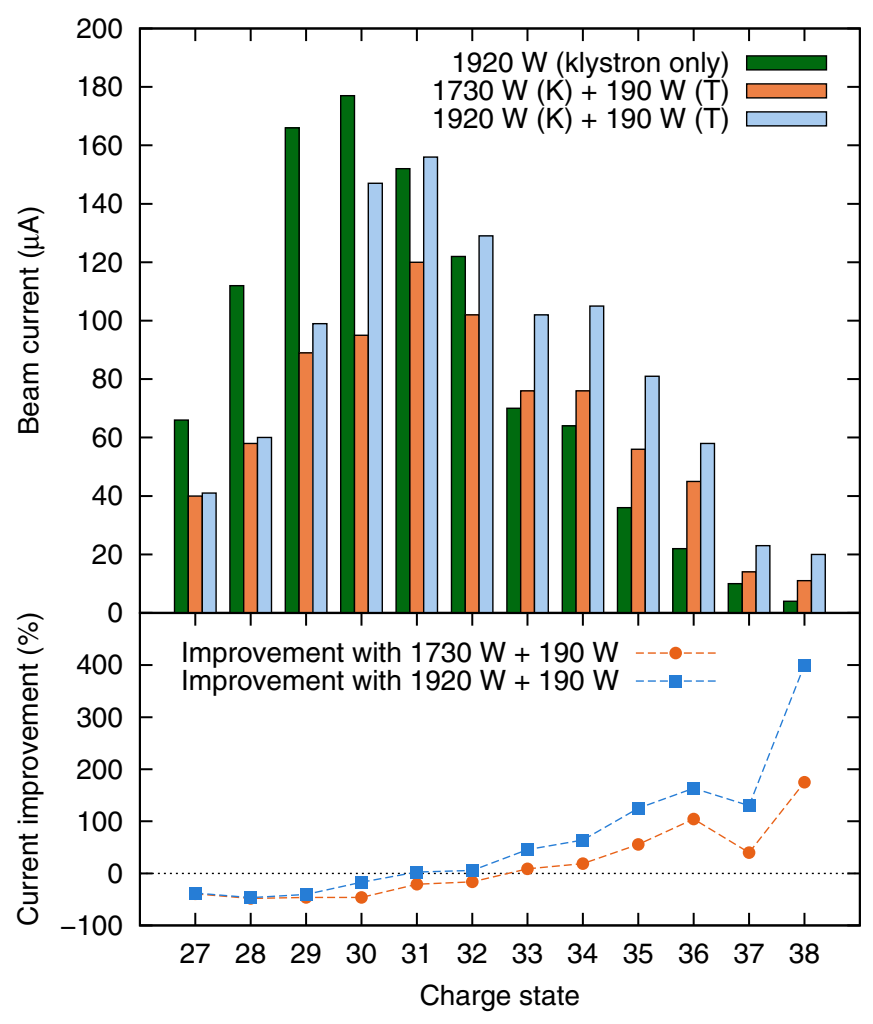

FIG. 11. Lead charge state distribution during afterglow with varied power balance between the two injected microwave frequencies. Klystron is operated at $14.50 \mathrm{GHz}$ and the TWTA at $12.36 \mathrm{GHz}$. Three cases are presented, (1) klystron only with nominal power level, (2) klystron and TWTA simultaneously with total power set to be comparable to the single frequency case, and (3) klystron and TWTA with increased total power. In addition the change in the beam current when using two frequencies instead of one is presented for the different charge states (points connected with dashed lines). 


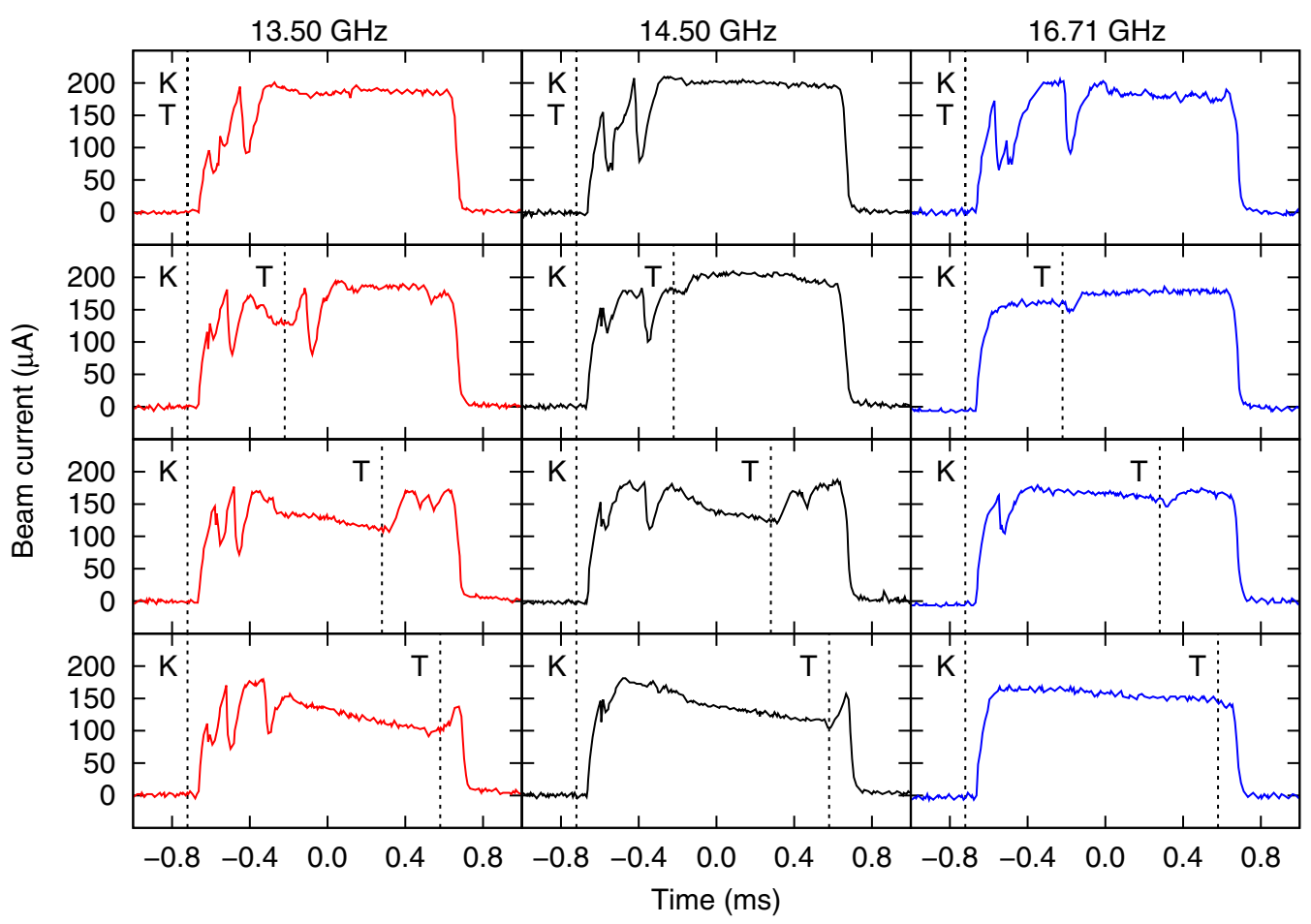

FIG. 12. ${ }^{208} \mathrm{~Pb}^{29+}$ afterglow beam currents with varied secondary frequency (columns) and trailing edge delays between the klystron and the TWTA RF pulses (rows). The presented delays are $0 \mathrm{~ms}$ for the first row, $0.5 \mathrm{~ms}$ for the second, $1.0 \mathrm{~ms}$ for the third and $1.3 \mathrm{~ms}$ for the fourth. The dashed vertical lines indicate the trailing edges of the klystron $(\mathrm{K})$ and the TWTA (T) RF pulses.

total power as in the first case, and (3) primary frequency at $1920 \mathrm{~W}$ and secondary at $190 \mathrm{~W}$. Figure 11 presents the measured charge state distributions associated with each of the cases. In both cases (2) and (3) the shift of the CSD to higher charge states is observed, indicating that the secondary frequency effect is not caused by the increase of the total power delivered by the two microwave sources. In addition, a further increase in the beam currents is seen when the total power is increased [case (3)].

The influence of the power at the secondary frequency on the afterglow was studied further by recording the beam current during the afterglow discharge with varied timing of the secondary frequency pulse compared to the primary microwave pulse. If both the primary and the secondary microwaves are switched off at the same moment, the beam current during the pulse varies as described above, but the shape of the afterglow pulse remains identical to the one acquired with single frequency operation. When a delay is applied to the switch-off of the secondary microwave pulse, it is observed that the afterglow current exhibits an additional step-like increase following the trailing edge of the secondary pulse. This effect is demonstrated with $\mathrm{Pb}^{29+}$ ion beam in Fig. 12 with selected secondary frequencies. When the delay is increased, a decreasing slope of the beam current is seen up to the time at which the secondary microwaves are switched off. This behavior differs from the relatively flat top that is normally associated with this part of the afterglow in the single frequency operation (or in the double frequency operation when both frequencies are switched off simultaneously). Consequently, the maximum beam current that is reached during the measured beam pulse is reduced when the delay time increases. Also, it is seen in Fig. 12 that the lower secondary frequencies have stronger impact on the shape of the beam pulse compared to the higher ones.

Comparing the beam current behavior of different charge states with a fixed klystron-TWTA trailing edge delay to operation with single frequency shows the same frequency dependent trends, which are seen in the charge state distributions presented in Fig. 10. With secondary frequencies lower than the primary one, the beam currents of the charge states lower than the peak of the CSD are reduced. Additionally, no clear second step is observed in the beam current following the switch-off of the secondary microwaves. For the charge states close to the peak of the distribution the beam current reaches the same or somewhat lower level as in the single frequency case after both microwave sources have switched off. The higher charge states exhibit comparable beam currents to the single frequency case after the trailing edge of the primary microwaves and are further increased after the trailing edge of the secondary microwaves. This behavior is demonstrated in the first column of Fig. 13 with $13.50 \mathrm{GHz}$ secondary frequency. When the secondary frequency is chosen to be higher than the primary one, as is the case with $16.70 \mathrm{GHz}$ in the second column of 


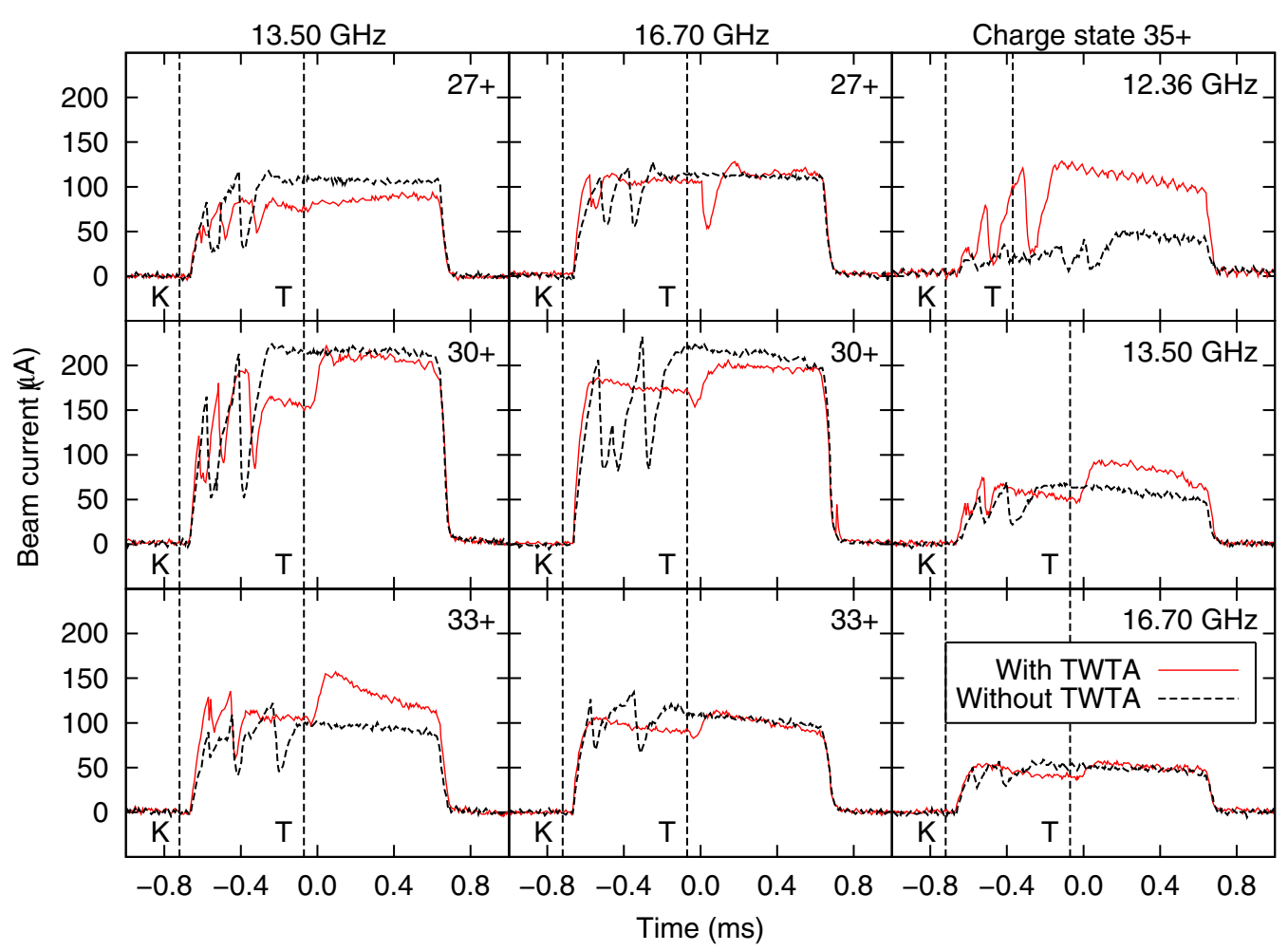

FIG. 13. Beam currents of selected lead ion charge states with single and double frequency heating. With double frequency heating a fixed delay of $0.65 \mathrm{~ms}\left(0.35 \mathrm{~ms}\right.$ for the $\mathrm{Pb}^{35+}$ at $12.36 \mathrm{GHz}$ case $)$ was used between the trailing edges of the primary $(\mathrm{K})$ and secondary (T) frequency pulses. The first and second columns presents charge states $27+, 30+$, and $33+$ with 13.50 and $16.70 \mathrm{GHz}$ secondary frequencies. The third column presents charge state $35+$ with frequencies $12.36,13.50$, and $16.70 \mathrm{GHz}$. The TWTA output power with the frequencies $12.36,13.50$, and $16.70 \mathrm{GHz}$ were 180,200 , and $185 \mathrm{~W}$, respectively.

Fig. 13, only minor differences are observed between the beam current levels of single and double frequency operation after both microwave sources have switched off, and no clear charge state dependence is seen. The strong frequency dependence of the beam current improvement of the highest charge states is further demonstrated in the last column of Fig. 13, which presents the $\mathrm{Pb}^{35+}$ beam currents with $12.36,13.50$, and $16.70 \mathrm{GHz}$ frequencies.

\section{DISCUSSION}

The experiments investigating the effect of double frequency heating in pulsed operation mode support the prevailing understanding of the afterglow physics but also raise new questions.

Based on the experimental results, operating the secondary microwave source in $\mathrm{CW}$ mode does not improve the afterglow beam currents of the high charge state ions. The lower (overall) beam currents with secondary frequency operated in CW mode suggests that part of the ion population, normally released in pulsed operation, is retained inside the plasma following the switch-off of the primary power source. This supports the commonly accepted qualitative explanation of the afterglow, which states that the burst of high charge state ions is a consequence of an abrupt increase of (cold) electron loss rate. Further evidence for this view is provided by the pulsed operation of the secondary frequency. When both microwave sources are pulsed, the observed improvement of the beam currents of highly charged lead ions during the afterglow is very similar to that observed in CW operation. R. Vondrasek et al. [6] have previously published a comparison of extracted lead beam currents with single and double frequency operation in $\mathrm{CW}$ mode for a $14 \mathrm{GHz}$ ECR ion source. An improvement of the high charge state currents was reported when the delivered power in double frequency mode was matched with the single frequency case, and a further increase was seen by increasing the total power with two frequencies, accompanied by a shift in the peak of the CSD up by two charge states. These results match the observed trends reported here for the afterglow operation. The two-stage behavior observed when varying the timing of the trailing edges of the two microwave pulses suggests that the plasma sustained by the secondary microwave radiation keeps a fraction of the electron (and ion) population trapped inside the chamber. Following the switch-off of the secondary frequency the remaining population escapes, resulting in the observed second increase of the extracted beam current. Thus the results imply that the mechanism causing the improved high 
charge state currents is occurring during the main rf pulse, i.e. linked to ionization and charge exchange processes.

The double frequency heating was observed to affect the high charge state production only when the secondary frequency is below the primary one, i.e. the secondary resonance zone is enclosed by the primary resonance. Furthermore, only the charge states higher than the peak of the CSD were clearly affected and exhibited steplike behavior when the relative timing of the rf pulses was shifted. This is consistent with the present understanding of spatial ion and plasma potential distributions in ECRIS plasmas $[20,21]$. The highly charged ions are believed to be trapped in a localized potential dip at the center of the plasma [22]. As the secondary frequency is switched-off the dip is presumably affected, resulting in the release of the trapped ion population, which is then observed as an increase in the extracted beam currents of high charge state ions. According to this qualitative view, secondary frequencies higher than the primary one should have a lesser impact on the ion confinement as suggested by the present data. Nevertheless, it is apparent that high secondary frequencies, which correspond to a resonance zone enclosing the primary resonance, do affect the electron energy distribution during the afterglow plasma decay. This is indicated by the observation that the afterglow instabilities, driven by the imbalance between hot and cold electron densities, vanished in $\mathrm{CW}$ operation of the secondary frequency above $14.5 \mathrm{GHz}$. A recent study has shown that this improvement in the temporal beam current stability is associated with the suppression of plasma instabilities by the secondary microwaves [23]. To our knowledge, this is the first time this stabilizing effect has been demonstrated with afterglow beam currents. In the $\mathrm{CW}$ studies the mitigation of instabilities has been reported when the secondary frequency was below the primary one. The experiments performed here do not offer an explanation for this observed discrepancy.

It is worth noting that the beneficial effect of the secondary microwave radiation was observed also at frequencies for which the cold electron resonance field is below $B_{\text {min }}$. This applies to both $\mathrm{CW}$ and pulsed operation of the secondary microwave source. Figure 14 presents the range of variation in the GTS-LHC axial $B$ field in the experiments around the minimum $B$ field value. The lowest value of the minimum- $B$ in the experiments was $0.47 \mathrm{~T}$. The magnetic field $B_{\mathrm{ECR}}$ at which the electron cyclotron resonance condition is fulfilled for microwaves of frequency $f_{\mathrm{mw}}$ can be described as [1]

$$
B_{\mathrm{ECR}}=\frac{2 \pi f_{\mathrm{mw}} \gamma m_{\mathrm{e}}}{e},
$$

where $e$ and $m_{\mathrm{e}}$ are the electron charge and mass and $\gamma$ is the relativistic gamma. For nonrelativistic electrons the lower limit for the microwave frequency to fulfill the resonance condition inside the plasma chamber is thus

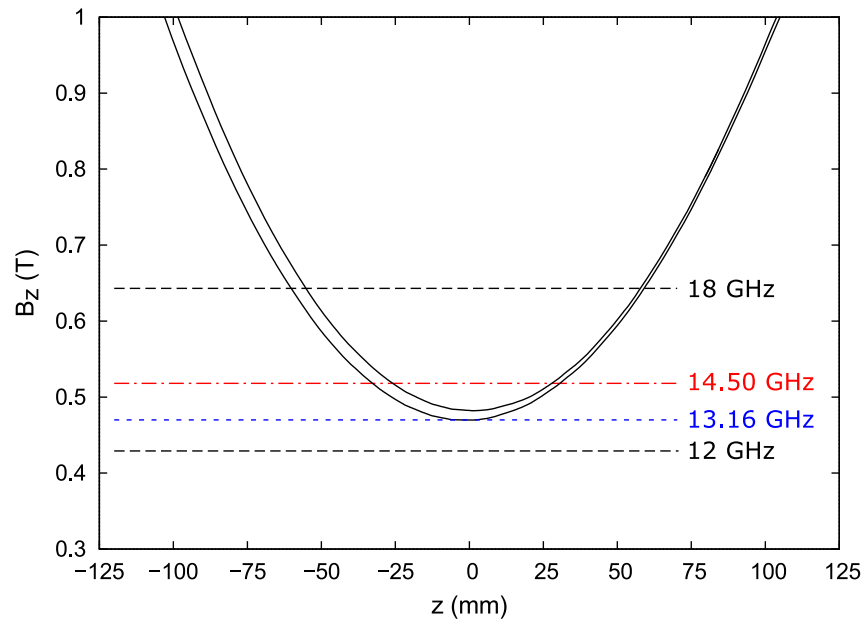

FIG. 14. The GTS-LHC magnetic field profile on axis around the minimum $B$ field and resonance fields for nonrelativistic electrons at selected frequencies. The magnetic fields resulting from the different solenoid settings used in the experiments fall within the presented field band.

13.16 GHz (see Fig. 14). Figure 9 indicates that the optimum secondary frequency for $\mathrm{Pb}^{35+}$ was approximately $13.5 \mathrm{GHz}$, i.e. the cold electron resonance field is very close to $B_{\min }$. However, also frequencies below $13.16 \mathrm{GHz}$ yield an improvement by a factor of $1.5-2$ for $\mathrm{Pb}^{35+}$. At high electron energies the relativistic mass increase of the electrons leads to higher values of resonance field $B_{\mathrm{ECR}}$, as is seen in Eq. (2). Following this, for the frequencies around $12.36 \mathrm{GHz}$ the resonance only exists for electrons with energies $>30 \mathrm{keV}$. Based on Ref. [24] for a given ion species the maximum of the electron impact ionization cross section is reached approximately at electron energies that are 3-4 times the ionization energy of the ion in question. Using the ionization energy data in Ref. [25] for lead ions, the cross section maxima for all the charge states measured in the experiments $(27+$ to $38+)$ is below $8 \mathrm{keV}$. Consequently, further heating of this electron population to higher energies (i.e. lower ionization cross sections) should not directly improve the ionization efficiency of the measured ion species. This implies that there are other indirect mechanisms which are responsible for the observed high charge state performance improvements. Furthermore, it has been reported recently that offresonance heating with secondary microwave frequency also resulted in performance improvements with $\mathrm{CW}$ operation [23]. In that study it was suggested that the observed effects were caused by suppression of cyclotron instabilities in the ECR plasma through the interaction of the secondary microwave radiation with the hot electron population. Another possibility could be improved ion confinement in the plasma through increased density of the well confined hot electron population. However, further studies are required to identify the exact mechanisms behind this observed behavior. 
Concerning the CERN operations, the principal lead beam delivered from the GTS-LHC for the experiments is currently ${ }^{208} \mathrm{~Pb}^{29+}$. As is seen in Fig. 9, the double frequency heating can provide only modest improvements for the beam current of this ion species. As the positive effects of this method are concentrated on the high end of the CSD, it is observed that ${ }^{208} \mathrm{~Pb}^{29+}$ is not a high enough charge state to benefit significantly from the secondary frequency. With additional fine tuning, a maximum increase of $10 \%-20 \%$ has been achieved in the ${ }^{208} \mathrm{~Pb}^{29+}$ beam current with $14.2 \mathrm{GHz}$ secondary frequency, as has been reported also in Ref. [26].

Altogether, the presented experimental results demonstrate the existence of the double frequency effect in the afterglow operation of an ECR ion source. As a result, this combined operation mode is a viable option to improve ion source performance in applications that require pulsed beams of highly charged ions.

\section{ACKNOWLEDGMENTS}

The authors would like to thank S. Bertolo, C. Mastrostefano and Francesco di Lorenzo for their technical assistance with the equipment used in the experiments and B. Woolley for his expertise in RF related issues.

[1] R. Geller, Electron Cyclotron Resonance Ion Sources and ECR Plasmas (IOP Publishing Ltd., London, 1996).

[2] G. Roland, K. Šafař́k, and P. Steinberg, Heavy-ion collisions at the LHC, Prog. Part. Nucl. Phys. 77, 70 (2014).

[3] D. Manglunki et al., CERN's fixed target primary ion programme, in Proceedings of the 7th International Particle Accelerator Conference, Busan, Korea, 2016.

[4] A. G. Drentje, Techniques and mechanisms applied in electron cyclotron resonance sources for highly charged ions, Rev. Sci. Instrum. 74, 2631 (2003).

[5] Z. Q. Xie and C. M. Lyneis, Two-frequency plasma heating in a high charge state electron cyclotron resonance ion source, Rev. Sci. Instrum. 66, 4218 (1995).

[6] R. Vondrasek, R. Scott, R. Pardo, H. Koivisto, O. Tarvainen, P. Suominen, and D. H. Edgell, ECRIS Operation With Multiple Frequencies, AIP Conf. Proc. 749, 31 (2005).

[7] G. Melin, F. Bourg, P. Briand, J. Debernardi, M. Delaunay, R. Geller, B. Jacquot, P. Ludwig, T. K. N'Guyen, L. Pin, M. Pontonnier, J.C. Rocco, and F. Zadworny, Some particular aspects of the physics of the ECR sources for multicharged ions, Rev. Sci. Instrum. 61, 236 (1990).

[8] F. Maimone, K. Tinschert, M. Endermann, R. Hollinger, S. Kondrashev, R. Lang, J. Mäder, P. T. Patchakul, and P. Spädtke, Investigation of pulsed mode operation with the frequency tuned CAPRICE ECRIS, Rev. Sci. Instrum. 87, $02 \mathrm{~A} 712$ (2016).

[9] L. Dumas, C. Hill, D. Hitz, D. Küchler, C. Mastrostefano, M. O'Neil, and R. Scrivens, Operation of the GTS-LHC source for the hadron injector at CERN, in Proceedings of the 17th International Workshop on ECR Ion Sources, Lanzhou, China, 2006.
[10] D. Hitz, D. Cormier, J. M. Mathonnet, A. Girard, G. Melin, F. Lansaque, K. Serebrenikov, and L. T. Sun, Grenoble Test Source (GTS): a multipurpose room temperature ECRIS, in Proceedings of the 15th International Workshop on ECR Ion Sources, Jyväskylä, Finland, 2002.

[11] C. E. Hill, D. Küchler, R. Scrivens, D. Hitz, L. Guillemet, R. Leroy, and J. Y. Pacquet, GTS-LHC: A New Source For The LHC Ion Injector Chain, AIP Conf. Proc. 749, 127 (2005).

[12] C. E. Hill, D. Küchler, C. Mastrostefano, M. O’Neil, R. Scrivens, D. Hitz, L. Guillemet, J. Chartier, J.-M. Mathonnet, and G. Rey-Giraud, Experience with the GTS-LHC ion source, in Proceedings of the 3rd LHC Project Workshop, Chamonix, France, 2006.

[13] O. Tarvainen, T. Ropponen, V. Toivanen, T. Kalvas, J. Ärje, and H. Koivisto, Diagnostics of plasma decay and afterglow transient of an electron cyclotron resonance ion source, Plasma Sources Sci. Technol. 19, 045027 (2010).

[14] D. Hitz, A. Girard, G. Melin, S. Gammino, G. Ciavola, and L. Celona, Results and interpretation of high frequency experiments at $28 \mathrm{GHz}$ in ECR ion sources, future prospects, Rev. Sci. Instrum. 73, 509 (2002).

[15] O. Tarvainen, T. Kalvas, H. Koivisto, J. Komppula, R. Kronholm, J. Laulainen, I. Izotov, D. Mansfeld, V. Skalyga, V. Toivanen, and G. Machicoane, Limitation of the ECRIS performance by kinetic plasma instabilities (invited), Rev. Sci. Instrum. 87, $02 \mathrm{~A} 703$ (2016).

[16] O. Tarvainen, T. Kalvas, H. Koivisto, J. Komppula, R. Kronholm, J. Laulainen, I. Izotov, D. Mansfeld, and V. Skalyga, Kinetic instabilities in pulsed operation mode of a $14 \mathrm{GHz}$ electron cyclotron resonance ion source, Rev. Sci. Instrum. 87, 02A701 (2016).

[17] D. Mansfeld, I. Izotov, V. Skalyga, O. Tarvainen, T. Kalvas, H. Koivisto, J. Komppula, R. Kronholm, and J. Laulainen, Dynamic regimes of cyclotron instability in the afterglow mode of minimum-B electron cyclotron resonance ion source plasma, Plasma Phys. Controlled Fusion 58, 045019 (2016).

[18] I. Izotov, T. Kalvas, H. Koivisto, J. Komppula, R. Kronholm, J. Laulainen, D. Mansfeld, V. Skalyga, and O. Tarvainen, Cyclotron instability in the afterglow mode of minimum-B ECRIS, Rev. Sci. Instrum. 87, 02A729 (2016).

[19] I. Izotov, D. Mansfeld, V. Skalyga, V. Zorin, T. Grahn, T. Kalvas, H. Koivisto, J. Komppula, P. Peura, O. Tarvainen, and V. Toivanen, Plasma instability in the afterglow of electron cyclotron resonance discharge sustained in a mirror trap, Phys. Plasmas 19, 122501 (2012).

[20] D. J. Clark, Emittance measurements on the LBL ECR source, in Proceedings of International Conference on ECR Ion Sources, East Lansing, USA, 1987.

[21] V. Mironov, S. Bogomolov, A. Bondarchenko, A. Efremov, and V. Loginov, Numerical model of electron cyclotron resonance ion source, Phys. Rev. ST Accel. Beams 18, 123401 (2015).

[22] V. P. Pastoukhov, Classical longitudinal plasma losses from open adiabatic traps, Rev. Plasma Phys. 13, 203 (1987).

[23] V. Skalyga, I. Izotov, T. Kalvas, H. Koivisto, J. Komppula, R. Kronholm, J. Laulainen, D. Mansfeld, and O. Tarvainen, Suppression of cyclotron instability in Electron Cyclotron Resonance ion sources by two-frequency heating, Phys. Plasmas 22, 083509 (2015). 
[24] The Physics and Technology of Ion Sources, edited by I. G. Brown (John Wiley \& Sons, Inc., New York, 2004).

[25] National Institute of Standards and Technology (NIST) Atomic Spectra Database, http://physics.nist.gov/ PhysRefData/ASD/ionEnergy.html.
[26] V. Toivanen, G. Bellodi, C. Fichera, D. Küchler, A. M. Lombardi, M. Maintrot, A. Michet, M. O’Neil, S. Sadovich, F. Wenander, and O. Tarvainen, Recent developments with the GTS-LHC ECR ion source at CERN, in Proceedings of the 22nd International Workshop on ECR Ion Sources, Busan, Korea, 2016. 